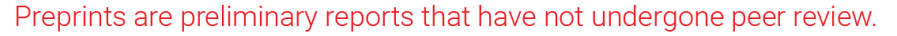 \\ They should not be considered conclusive, used to inform clinical practice, or referenced by the media as validated information. \\ The Ribosomal Protein L28 Gene Is Involved in Sorafenib Resistance in Hepatocellular Carcinoma
}

\section{Yi Shi}

Fujian Provincial Cancer Hospital

\section{Xiaojiang Wang}

Fujian Provincial Cancer Hospital

\section{Qiong Zhu}

Fujian Medical University

Gang Chen ( $\nabla$ naichengang@126.com )

Fujian Provincial Cancer Hospital

\section{Research}

Keywords: Ribosomal protein L28 (RPL28) Gene, HCC, Sorafenib resistance, Molecular mechanism

Posted Date: March 16th, 2021

DOI: https://doi.org/10.21203/rs.3.rs-259580/v1

License: (9) This work is licensed under a Creative Commons Attribution 4.0 International License. Read Full License 


\section{Abstract}

Background: Sorafenib is the first molecular-targeted drug for the treatment of advanced hepatocellular carcinoma (HCC). However, its treatment efficiency decreases after a short period of time because of the development of drug resistance. This study investigates the role of key genes in regulating sorafenibresistance in hepatocellular carcinoma and elucidates the mechanism of drug resistance.

Methods: The HCC HepG2 cells were used to generate a sorafenib-resistant cell model by culturing the cells in gradually increasing concentration of sorafenib. RNA microarray was applied to profile gene expression and screen key genes associated with sorafenib resistance. Specific targets were knockdown in sorafenib-resistant HepG2 cells for functional studies. The HCC model was established in ACl rats using Morris hepatoma3924A cells to validate selected genes associated with sorafenib resistance in vivo.

Results: The HepG2 sorafenib-resistant cell model was successfully established. The $\mathrm{IC}_{50}$ of sorafenib was $9.988 \mathrm{mM}$ in HepG2 sorafenib-resistant cells. A total of 35 up-regulated genes were detected by expression profile chip. High-content screening technology was used and a potential drug-resistant gene $R P L 28$ was filtered out. After knocking down of RPL28 in HepG2 sorafenib-resistant cells, the results of cell proliferation and apoptosis illustrated that RPL28 is the key drug-resistant gene in the cells. Furthermore, it was found that both RNA and protein expression of RPL28 increased in HepG2 sorafenibresistant specimens of Morris Hepatoma rats. In addition, the expression of functional proteins Ki-67 increased in sorafenib-resistant cells.

Conclusion: Our study suggested that $R P L 28$ was a key gene for sorafenib resistance in HCC both in vitro and in vivo.

\section{Background}

In recent years, a number of tyrosine kinase inhibitor drugs, such as sorafenib, regorafenib, and lenvatinib have been approved in China and have become the main treatment options for patients with advanced liver cancer. Sorafenib is the first molecular-targeted drug approved to treat advanced hepatocellular carcinoma (HCC). Sorafenib, named as Dodgemet in the market, targets RAF kinase, VEGFR1-3 and PDGF receptors. Shingina et al. and Kudo et al. reported that the median survival time, disease control rate, median imaging progression time and overall survival time are better in sorafenib treated patients than those treated with placebo [1]. Chung et al. reported that HCC patients treated with sorafenib combined with TACE achieved similar results in Chinese subgroup [2]. Regorafenib is another molecular-targeted tyrosine kinase inhibitor. LeBerre et al. confirmed that regorafenib could extend the median overall survival time by 2.8 months and the disease control rate reaching $65 \%$ in HCC patients who progressed after sorafenib treatment,[3]. Currently, several cancer treatment guidelines have been updated: regorafenib could be used to treat advanced liver cancer after sorafenib treatment. Lenvatinib is the latest oral tyrosine poly-kinase inhibitor. Kudo et al. compared the therapeutic effect of lenfatinib and sorafenib 
as a first-line treatment regimen for inoperable liver cancer patients. They found that the median overall survival time and objective response rate of lenfatinib is significantly better than that of sorafenib [4], suggesting that lenfatinib might be a new first-line treatment option for patients with advanced HCC.

Although these kinases inhibitor drugs bring hopes for liver cancer patients, there are also problems in clinical application. In HCC patients who have severe chronic liver disease, the impact of survival benefits has not been confirmed due to drug-induced liver toxicity $[5,6]$. Furthermore, there are still major challenges such as lack of effective biomarkers to predict drug efficacy, low objective drug response rate, non-significant tumor burden reduction and poor quality of life of patients [7]. In addition, most patients develop drug resistance in the treatment process. Although sorafenib was proved as a well-established molecular targeted drug for HCC therapy, the cancer inhibition efficiency decreases in most patients after an average of 17.6 weeks and the mechanism of drug resistance is not clear. Because sorafenib, regarafenib and lenvatinib are multiple target drugs, their mechanism of action involves multiple signaling pathways and involves both aspect of tumor cells and tumor microenvironment, therefore, their drug resistance mechanisms are complex [8]. Due to the high heterogenicity of HCC, the same tumor tissue might have different HCC cell subsets. Different sorafenib resistance mechanisms might occur in the same patient during treatment process and effective molecular biomarkers are needed in clinical application.

Ribosomal protein L28 (RPL28) gene is located on human chromosome 19q13.42 and encode the 60S large subunit components of ribosomal protein L28E family [9]. Ribosome is the place for protein synthesis, involving the basic functions of protein synthesis, DNA replication, RNA splicing and translation [10]. Fan et al. reported that silencing ribosomal protein L28 could inhibit the proliferation and invasion of esophageal cancer cells $[11,12]$. Li et al. reported that the growth of human pancreatic cancer cells could be inhibited by RNA interference down-regulated ribosomal protein L39 [13]. Hide et al, observed the difference in the expression of RPL28 in colorectal cancer and adjacent tissues, but no relevance was found between the expression of L28 and development of colorectal cancer $[14,15]$.

This study aims to investigate the cause of sorafenib resistance. We found that RPL28 might be a key gene of sorafenib resistance in HCC.

\section{Materials And Methods}

\section{Establishing the sorafenib resistant cell model of hepatocellular carcinoma}

We established the sorafenib (Selleck) acquired drug resistant HepG2 cell line by culturing HepG2 cells with gradually increasing concentrations of sorafenib. Before the experiment, cell viability assay was conducted by Cell Titer-Glo Luminescent (Promega) and $\mathrm{IC}_{50}$ of sorafenib in HepG2 cells was selected as the starting drug concentration. Then, cells were maintained for one week until they grew steadily. After that, increased concentration of sorafenib $(0.25 \mathrm{~mol} / \mathrm{L}$ each time $)$ was added to the culture medium. The 
induction process lasted for 6 months when the cells could grow steadily at the maximum tolerable concentration of sorafenib. Finally, the sorafenib resistant cell model of HepG2 was successfully constructed.

\section{Screening drug resistance genes}

Gene chip Primeview human (Affymetrix) for expression spectrum profiling was applied to select the drug resistance-related genes. Screening criteria were fold change $(F C)>2$ and $P$-value $<0.05$. Stratagene Mx3000P real-time PCR (Agilent) was used to detect the endogenous expression of drug resistancerelated genes in cells.

The key resistance gene RPL28 was screened by High Celigo Select (HCS) technique.

The characteristic of drug-resistant cells is the loss of response to drug. Therefore, we observed the effect of silencing target gene on cell proliferation by down regulating the expression of target gene in lentivirus infected cells. The green fluorescent protein GFP was expressed by infected cells and counted automatically. In order to ensure the gene interference efficiency, we designed three RNA interference targets for each gene, and we take three plasmids with different targets mixed as equal proportion and then packaged with lentivirus, to make sure knockout efficiency of target gene in the subsequent HCS experiment after the virus infected cells. Cells were photographed and automatically counted by the Celigo fluorescence microscope (Olympus). The influence of the cell proliferation for each gene was detected by the high throughput model.

By the same method, we screen out the target with the most significant inhibition of cell proliferation and the highest knockout efficiency of key resistance gene. After determining the proliferation-related positive target genes, lentivirus packaging and HCS cell proliferation detection were performed on the plasmids of the three RPL28 RNA interference targets, to further confirm the specific targets of the gene affecting the cell proliferation.

Using RPL28-1 target as a template(Table 1), we prepared RNA interference lentivirus vectors and infected HepG2 sorafenib resistant cells. The reduced protein expression of $R P L 28$ gene was detected by Western blot. The effect of RPL28 gene on the proliferation, apoptosis and cell cycle of HepG2 sorafenib resistant cells were detected by Celigo (Nexcelom), Caspase-Glo ${ }^{\circledR} 3 / 7$ Assay (Promega), Apoptosis kit (BD) and PI (Sigma)

Table 1

RNAi target sequence

\begin{tabular}{|ll|}
\hline Gene targets & Sequences \\
\hline$R P L 28-1$ & CTACAGCACTGAGCCCAATAA \\
\hline$R P L 28-2$ & TGGTGGTCATTAAGCGGAGAT \\
\hline$R P L 28-3$ & CCGCAATTCCTTCCGCTACAA \\
\hline
\end{tabular}




\section{Morris Hepatoma Rat Model for sorafenib resistance}

SPF rats were used to establish Morris hepatoma liver cancer model. Morris hepatoma 3924A (MH3924A) cells were subcutaneously injected and about $1 \times 1 \times 1 \mathrm{~cm}^{3}$ tumor could be touchable in the subcutaneous in about 2 weeks. Under aseptic conditions, the tumor bulk was inoculated to the left lobe of the liver of $\mathrm{ACl}$ rats to create liver carcinoma model in situ. After treatment with sorafenib, dynamic change of rat tumor in sensitive period and resistance period of sorafenib were detected by PET-CT. The expression of RPL28 gene in sorafenib-resistant HCC was tested by Real time qPCR. IHC was used to detect the expression of $\mathrm{KI}-67$ in samples in both sorafenib sensitive and resistance stage. The intensity and the proportion of stained cells were integrated, and the result was determined by semi-quantitative integral method using the following scoring criteria: staining intensity score, $0=$ no staining, $1=$ weak staining, 2 = moderate staining, 3 = strong staining; staining percentage score, $0=$ positive percentage $<$ $10 \%, 1=10-25 \%, 2=26-50 \%, 3=51-75 \%, 4>75 \%$. The staining score $=$ intensity score $x$ percentage. The score of $0-3$ indicates low expression, while 4-7 indicates high expression.

\section{Results}

\section{Testing the sorafenib resistant cell line}

The cell proliferation in response to increasing concentrations of sorafenib was examined in both HepG2 control and HepG2 drug resistant cells. It was found that the inhibitory rate of HepG2 cells increased significantly with the increasing drug concentrations, with the $\mathrm{IC}_{50}$ of sorafenib being $1.62 \mu \mathrm{M}$ in control cells, while no significant change on inhibitory rate in HepG2 drug-resistant cells, and the $\mathrm{IC}_{50}$ of $9.988 \mu \mathrm{M}$. This result indicated the successful establishment of sorafenib resistant cell model (Fig. 1).

\section{Detecting drug resistant genes}

Gene expression profile chip was used to screen genes related to drug resistance. 295 up-regulated genes and 211 down-regulated genes were found in HepG2 sorafenib resistant cells compared with HepG2 control cells (Fig. 2A). Through the bioinformatics analysis, 35 up-regulated genes were selected for further study based on their highly expressed profiles in drug-resistant cells.

Among the 35 drug-resistant related genes, two genes were not detected by qPCR due to difficulty in primer design, and one gene had low expression. The remaining 32 drug-resistant related genes had high abundance of expression. Twenty genes were randomly selected based on the screening condition with proliferation change $\geq 2.0$, and the difference significance analysis T-test value $<0.05$. The key drugresistant gene RPL28 was selected by comparing the effects of gene knockdown on cell proliferation using HCS technology. RPL28-1 was finally selected as the key drug-resistant gene. After knocking-down of RPL28-1, the proliferation inhibition of HepG2 drug-resistant cells reached 2.76 times compared with the control cells, and the knockdown efficiency reached $86.1 \%$ compared with the control cells (Fig. 2B), 
and the difference was statistically significant $(P<0.05)$, indicating that RPL28-1 was indeed an effective target.

\section{Preparing RNAi lentiviral vector of RPL28 gene and RPL28 knockdown affected protein expression}

Using RPL28 gene as a template, RNA interference target sequence was designed, and RNA interfering lentivirus vector was constructed. The sequence of RPL28 gene interference target in the vector starts from the $\mathrm{C}$ of $383 \mathrm{bp}$ and ends at $\mathrm{A}$ of $403 \mathrm{bp}$ (Fig. 3A). HepG2 drug-resistant cells were infected by lentiviruses with infection efficiency of above $80 \%$, indicating that the RPL28 knockdown sorafenib resistant HepG2 cell line was successfully established. Western Blot was applied to detect the expression of exogenous protein of RPL28 gene and we found that knockdown the RPL28-1 target affected the exogenous expression of RPL28 gene (Fig. 3B), which further proved that RPL28-1 was an effective interference target.

\section{Biological function study of HepG2 sorafenib resistant cells infected by shRNA lentivirus}

After knocking- down of RPL28 gene, the proliferation of sorafenib resistant HepG2 cells was significantly inhibited as tested by Celigo. Cell counting result showed that the proliferation of $\operatorname{sh} R L 28$ cells was significantly inhibited compared with the shCtrl cells (Fig. 4A). Caspase-Glo® 3/7 Assay and flow cytometric analysis showed that the apoptosis rate of sorafenib resistant HepG2 cells increased when RPL28 gene was knockdown (Fig. 4B). The results of cell cycle analysis illustrated that the cells in $\mathrm{S}$ phase increased after knocking-down RPL28 in HepG2 sorafenib resistant cells and decrease the cell number in $\mathrm{G} 1$ phase. Our results showed the strong association between the function of RPL28 and cell cycle change in HepG2 sorafenib resistant cells (Fig. 4C).

\section{In vivo evaluation for sorafenib resistance}

Dynamic changes of tumor in rat with sorafenib treatment were evaluated by PET-CT. The tumor was controlled in the sorafenib sensitive stage (Fig. 5A), however, during sorafenib resistant stage, the tumor can be seen extensively metastasis to abdominal cavity (Fig. 5B).

The expression of sorafenib-resistant associated gene RPL28 in Morris Hepatoma was determined by real time qPCR. The expression of RPL28 increased in the sorafenib-resistant specimens compared with that in sensitive phase with statistically significant $(\mathrm{P}<0.05)$, indicating that $R P L 28$ gene was associated with sorafenib resistance. The expression of Ki-67 in HCC tissues of rats with sorafenib sensitive stage was lower than that of rats with drug resistance stage, indicating that sorafenib inhibited the proliferation of HCC cells (Fig. 6A \& 6B).

\section{Discussion}


The $R P L 28$ gene is the typical gene which encodes ribosomal protein with many pseudo-genes scattered throughout the genome and different splicing transcription forms. In this study, the expression of RPL28 gene was found to be significantly increased in sorafenib resistant HepG2 cells compared with parental HepG2 cells. Ribosomes act as a processing factory of protein polypeptide chains and the expression of $\mathrm{RP}$ itself reflects the state of cell growth. Ribosome proliferation also indicates proliferative activity of both normal and tumor tissues. Growing cells have more efficient ability for translating RP mRNA than stationary cells. Thus, cells with proliferating activity usually have high RP levels. Therefore, when HepG2 cells developed resistance to sorafenib, the cell proliferation inhibited due to drug action became active again, and the expression of ribosomal protein increased accordingly, which could explain our experimental results.

In order to verify the experimental results, we constructed a RPL28 RNA interference lentiviral vector, and the HepG2 sorafenib resistant cells were infected with the lentivirus to down-regulate the RPL28 gene expression. It was found that knocked out of $R P L 28$ gene significantly inhibited the proliferation of HepG2 sorafenib resistant cells and increased apoptotic cells. Our results suggested that interfering RPL28 gene could inhibit the proliferation of HepG2 sorafenib resistant cells, reversing sorafenib resistance. Therefore, up-regulation of $R P L 28$ gene might be one of the causes of sorafenib resistance, and its mechanism is associated with the effect of RP in tumors. Changes in RP expression may affect the protein translation process. Both the assembly and function of ribosomes depend on the balance of the quantity of RP. The increased ribosomes may accelerate the translation of ribosomes and affect the synthesis of proteins that affect the metabolism and biological functions of cells. We speculated that the increased RPL28 gene expression would accelerate mRNA translation and protein synthesis, promote HepG2 cell proliferation, and lead to sorafenib resistance.

Ribosomal proteins can regulate cell apoptosis. Zhang et al. reported that RP S15A was highly expressed in glioma compared with normal tissues. Down-regulation of RPS15A could induce apoptosis of glioma cells [16]. Our results indicated that the increased expression of RPL28 gene might down-regulate certain apoptotic proteins, inhibit the programmed cell death of HepG2 cells, and result in sorafenib resistance. The most reported function of ribosomal protein is related to tumor suppressor gene P53. Peng et al. reported that ribosomal protein L23 inhibit P53 ubiquitination by negatively regulating murine double minute 2 (MDM2), thereby activating P53 to inhibit tumor growth $[17,18]$. It might be possible that the increased expression of $R P L 28$ gene also regulate the P53 gene, and lead to sorafenib resistance.

Morris hepatoma3924A is a highly metastatic cell strain of rat liver cancer. Due to its high tumor formation rate in vivo, it has been widely used to establish various animal models. Jun et al. applied Morris hepatoma3924A in ACl rat to explore nanoscale integrin combined with arterial chemoembolization for the treatment of liver carcinoma [19]. In this study, Morris hepatoma3924A in ACI rat model was used to study sorafenib resistance in vivo. It was found that the tumor growth was effectively controlled in the sorafenib sensitive stage. The tumor had extensive metastasis in the abdominal cavity in the sorafenib resistant stage. 
Real time GPCR and IHC results showed that the protein expression of $R P L 28$ gene increased in the resistant phase compared with the sensitive phase, which is in consistent with the results in vitro, and indicating that $R P L 28$ gene might be the sorafenib-resistant gene in vivo. In addition, immunohistochemical staining results showed that Ki-67 protein expression increased in the drugresistant stage compared to the sensitive stage. $\mathrm{Ki}-67$ is used to judge cell proliferative activity. It is expressed in all cell cycles (G1, S, G2 and M stages), but not expressed in G0 stage. Previous studies indicated that the $\mathrm{KI}-67$ expression is closely associated with cell proliferation, differentiation, invasion and metastasis, and prognosis of various tumors. In this study, ki-67 protein expression increased in sorafenib resistance rats, suggesting that sorafenib resistance is related to cell proliferation. The cell cycle regulatory core molecule is CDK, which is dependent on the expression of Cyclin. CDK4 is one of the CDK series of protein kinases. CDK4 kinase activity is activated when CDK4 binds to periodic protein D1 (cyclin D1) to form the CDK4 / cyclin D1 complex, which phosphorylates Retinoblastoma protein (Rb). Rb protein releases a transcription factor $E 2 F$, results in the expression of $S$ phase related proteins induced by E2F. It promotes cells to complete DNA replication, quickly across the G1 to S phase limit point, stimulate cell growth and division [20]. Over-expression of CDK4 caused HepG2 cells growth out of control, promoted cell proliferation and abnormal differentiation leading to sorafenib resistance. Bcl-2 (Bcell lymphoma 2 gene) is an oncogene. Its family of proteins play an important role in mitochondrial apoptosis. The $\mathrm{Bcl}-2$ protein family has high homology and contains $\mathrm{BH}(1-4)$ conserved domains. The function of Bcl-2 protein family can be divided into two categories, one is anti-apoptotic: including Bcl-2, $\mathrm{Bcl}-\mathrm{XL}$ and $\mathrm{McL}-1$, that are located in the mitochondrial outer membrane, endoplasmic reticulum and nuclear membrane. The other is apoptotic promoting, including Bax, Bak and Bid. They are mostly located in the cytoplasm [21].

\section{Conclusion}

In conclusion, this study established a sorafenib resistant hepatocellular carcinoma cell mode. The drug resistant gene was screened by expression profile chip technology and confirmed by experiments both in vitro and in vivo. We suggested that $R P L 28$ gene is involved in sorafenib resistance in hepatocellular carcinoma. Our study provided a new approach to overcome sorafenib resistance in the treatment of hepatocellular carcinoma.

\section{Abbreviations}

Bcl-2

B-cell lymphoma 2; GFP:green fluorescent protein; HCC:hepatocellular carcinoma; MH3924A:Morris

hepatoma 3924A; RPL28:Ribosomal protein L28; HCS:High Celigo Select; PCR:polymerase chain reaction

\section{Declarations}

Ethics approval and consent to participate: This study was approved by the Fujian Medical University Cancer Hospital 


\section{Consent for publication: N/A}

Availability of data and material: All data and material will be available upon request by writing to the corresponding author.

Competing interests: Authors declare no competing interest

Funding: This research was supported by the Natural Science Foundation of Fujian Province, No. 2018J01268; Fujian Provincial Health Technology Project, No. 2018-ZQN-15; and Science and Technology Program of Fujian Province, China, Nos. 2018Y2003, 2019L3018, and 2019YZ016006.

Authors' contributions: GC conceived and designed the study. YS, XW and QZ performed experiments and analyzed results. YS wrote the first draft. GC edited the manuscript. All authors read and approved the final manuscript.

\section{Acknowledgment: N/A}

\section{References}

1. Shingina A, Hashim A, Haque M, Suen MW, Yoshida EM, Gill S. and A. Weiss, 171 Sorafenib for Advanced Hepatocellular Carcinoma. Gastroenterology 142 (2012).

2. Chung YH, Han G, Yoon JH, Yang J, Wang J, Shao GL, Kim BI, Lee TY, Chao Y. Interim analysis of START: Study in Asia of the combination of TACE (transcatheter arterial chemoembolization) with sorafenib in patients with hepatocellular carcinoma trial. Int J Cancer. 2013;132:2448-58.

3. Bruix J, Qin S, Merle P, Granito A, Huang Y-H, Bodoky G, Pracht M, Yokosuka O, Rosmorduc O, Breder V, Gerolami R, Masi G, Ross PJ, Song T, Bronowicki J-P, Ollivier-Hourmand I, Kudo M, Cheng A-L, Llovet JM, Finn RS, LeBerre M-A, Baumhauer A, Meinhardt G, Han G. Regorafenib for patients with hepatocellular carcinoma who progressed on sorafenib treatment (RESORCE): a randomised, doubleblind, placebo-controlled, phase 3 trial. The Lancet. 2017;389:56-66.

4. Kudo M, Finn RS, Qin S, Han K-H, Ikeda K, Piscaglia F, Baron A, Park J-W, Han G, Jassem J, Blanc JF, Vogel A, Komov D, Evans TRJ, Lopez C, Dutcus C, Guo M, Saito K, Kraljevic S, Tamai T, Ren M, Cheng A-L. Lenvatinib versus sorafenib in first-line treatment of patients with unresectable hepatocellular carcinoma: a randomised phase 3 non-inferiority trial. The Lancet. 2018;391:1163-73.

5. de Rosamel L, Blanc JF. Emerging tyrosine kinase inhibitors for the treatment of hepatocellular carcinoma. Expert Opin Emerg Drugs. 2017;22:175-90.

6. Sun J, Cai X, Yung MM, Zhou W, Li J, Zhang Y, Li Z, Liu SS, Cheung ANY, Ngan HYS, Li Y, Dai Z, Kai Y, Tzatsos A, Peng W, Chan DW, Zhu W. miR-137 mediates the functional link between c-Myc and EZH2 that regulates cisplatin resistance in ovarian cancer. Oncogene. 2019;38:564-80.

7. Hsu CY, Liu PH, Ho SY, Huang YH, Lee YH, Chiou YY, Hsieh TH, Fang T, Tsai YJ, Hou MC, Huo TI. Impact of tumor burden on prognostic prediction for patients with terminal stage hepatocellular carcinoma: A nomogram study. PLoS One. 2017;12:e0188031. 
8. Zhu YJ, Zheng B, Wang HY, Chen L. New knowledge of the mechanisms of sorafenib resistance in liver cancer. Acta Pharmacol Sin. 2017;38:614-22.

9. Goudarzi KM, Lindstrom MS. Role of ribosomal protein mutations in tumor development (Review). Int J Oncol. 2016;48:1313-24.

10. Warner JR, Mclntosh KB. How common are extraribosomal functions of ribosomal proteins? Mol Cell. 2009;34:3-11.

11. Hirashita Y, Tsukamoto Y, Yanagihara K, Fumoto S, Hijiya N, Nakada C, Uchida T, Matsuura K, Kodama M, Okimoto T, Daa T, Seike M, Iha H, Shirao K, Murakami K, Moriyama M. Reduced phosphorylation of ribosomal protein $\mathrm{S} 6$ is associated with sensitivity to MEK inhibition in gastric cancer cells. Cancer Sci. 2016;107:1919-28.

12. Fan H, Li J, Jia Y, Wu J, Yuan L, Li M, Wei J, Xu B. Silencing of Ribosomal Protein L34 (RPL34) Inhibits the Proliferation and Invasion of Esophageal Cancer Cells. Oncol Res. 2017;25:1061-8.

13. Li C, Chen D, Luo M, Ge M, Zhu J. Knockdown of ribosomal protein L39 by RNA interference inhibits the growth of human pancreatic cancer cells in vitro and in vivo. Biotechnol J. 2014;9:652-63.

14. Kasai H, Nadano D, Hidaka E, Higuchi K, Kawakubo M, Sato TA, Nakayama J. Differential expression of ribosomal proteins in human normal and neoplastic colorectum. J Histochem Cytochem. 2003;51:567-74.

15. Zhang Y, Li Z, Hao Q, Tan W, Sun J, Li J, Chen CW, Li Z, Meng Y, Zhou Y, Han Z, Pei H, DePamphilis ML, Zhu W. The Cdk2-c-Myc-miR-571 Axis Regulates DNA Replication and Genomic Stability by Targeting Geminin. Cancer Res. 2019;79:4896-910.

16. Zhang C, Fu J, Xue F, Ryu B, Zhang T, Zhang S, Sun J, Xu X, Shen Z, Zheng L, Chen X. Knockdown of ribosomal protein S15A induces human glioblastoma cell apoptosis. World $\mathrm{J}$ Surg Oncol. 2016;14:129.

17. Liu Y, Deisenroth C, Zhang Y. RP-MDM2-p53 Pathway: Linking Ribosomal Biogenesis and Tumor Surveillance. Trends Cancer. 2016;2:191-204.

18. Sun J, Fu X, Wang Y, Liu Y, Zhang Y, Hao T, Hu X. Erianin inhibits the proliferation of T47D cells by inhibiting cell cycles, inducing apoptosis and suppressing migration. Am J Transl Res. 2016;8:307786.

19. Qian J, Oppermann E, Tran A, Imlau U, Qian K, Vogl TJ. Transarterial administration of integrin inhibitor loaded nanoparticles combined with transarterial chemoembolization for treating hepatocellular carcinoma in a rat model. World J Gastroenterol. 2016;22:5042-9.

20. Li Z, Zhou W, Zhang Y, Sun W, Yung MMH, Sun J, Li J, Chen CW, Li Z, Meng Y, Chai J, Zhou Y, Liu SS, Cheung ANY, Ngan HYS, Chan DW, Zheng W, Zhu W. ERK Regulates HIF1alpha-Mediated Platinum Resistance by Directly Targeting PHD2 in Ovarian Cancer. Clin Cancer Res. 2019;25:5947-60.

21. Camperchioli A, Mariani M, Bartollino S, Petrella L, Persico M, Orteca N, Scambia G, Shahabi S, Ferlini $\mathrm{C}$, Fattorusso $\mathrm{C}$. Investigation of the Bcl-2 multimerisation process: structural and functional implications. Biochim Biophys Acta. 2011;1813:850-7. 
Figures

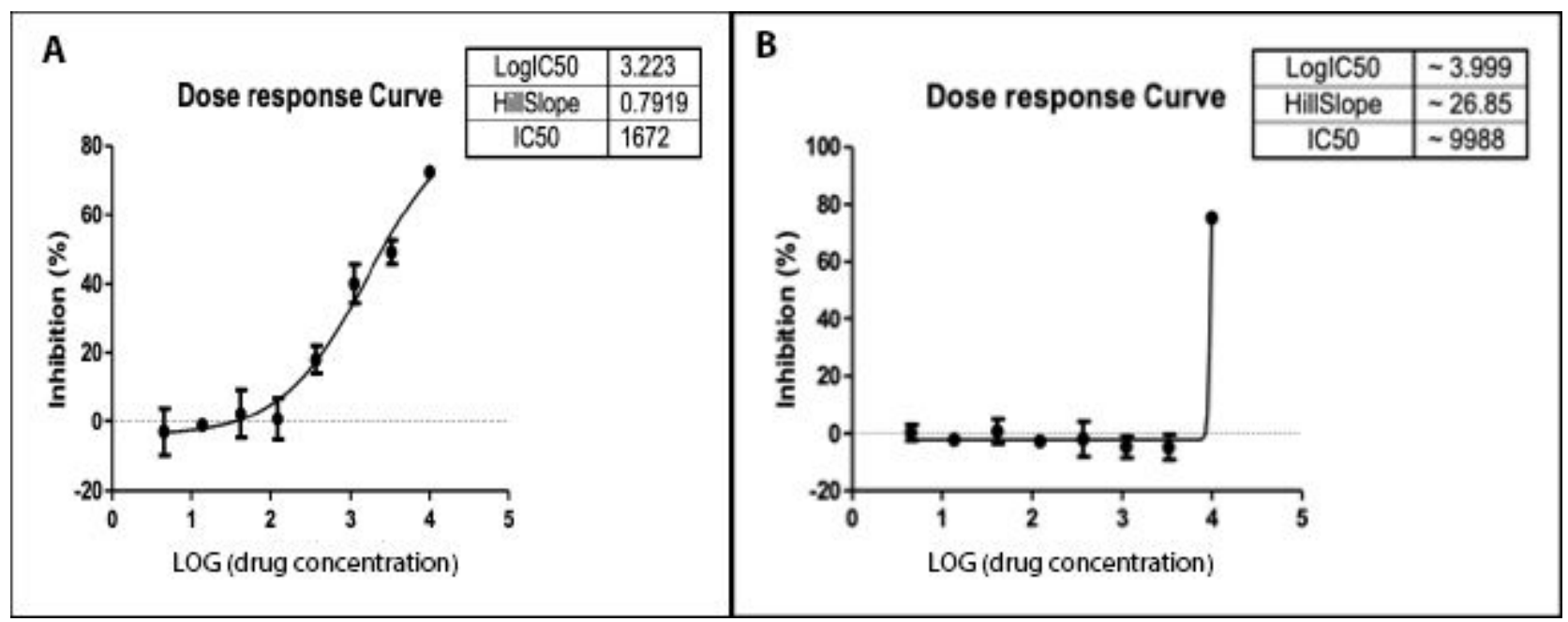

Figure 1

Inhibition curves of sorafenib on HepG2 cells. A. The inhibitory rate of HepG2 cells increased with the increasing drug concentrations; B No significant change on inhibitory rate of HepG2 drug-resistant cells.
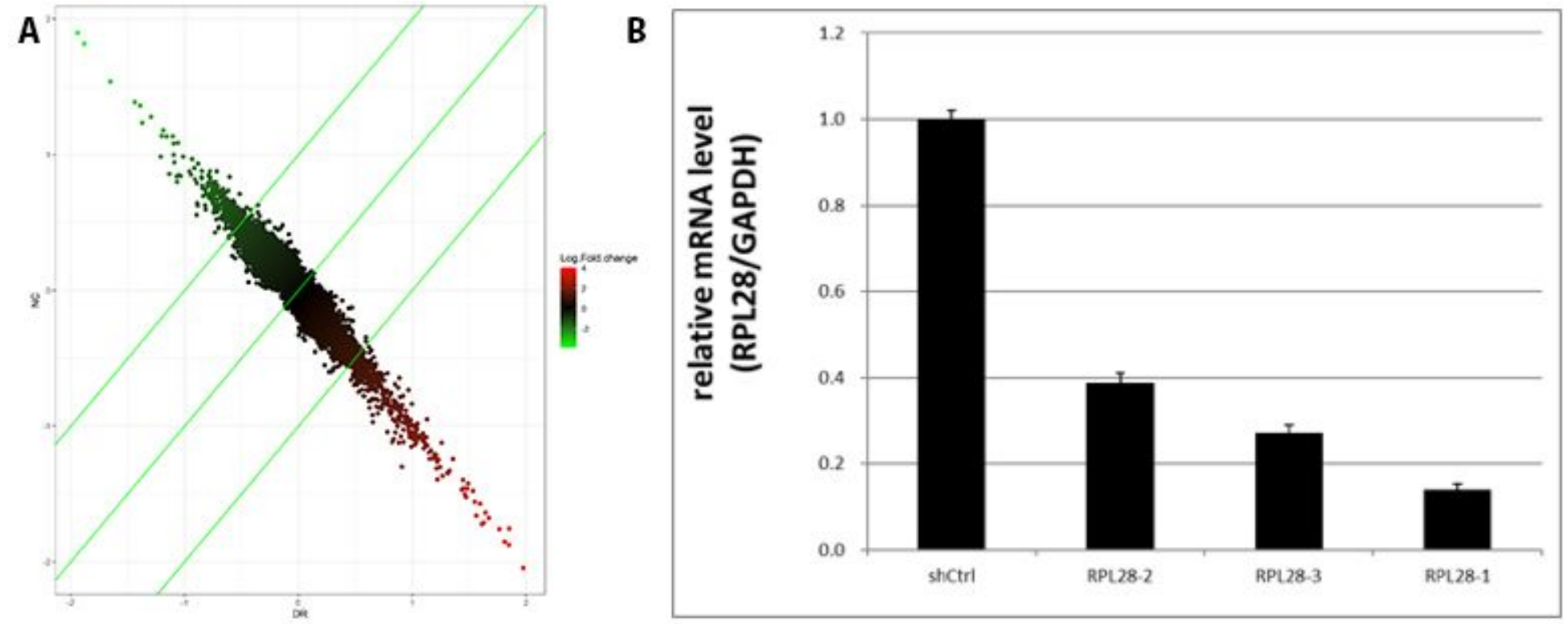

Figure 2

A. Drug-resistant related genes detected by microarray. B. Expression of RPL28 gene after it was knocked down. The expression of RPL28 genes in HepG2 drug-resistant cells was 0.139,0.389 and 0.272, compared with control group, after RPL28-1, RPL28-2 and RPL28-3 target was knocked down, respectively. The differences were statistically significant $(P<0.05)$ 


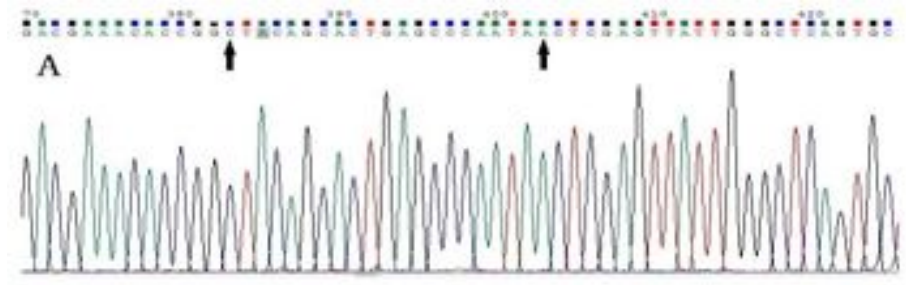

B

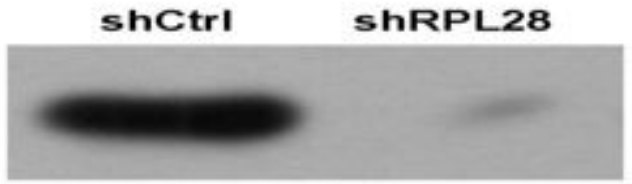

GAPDH

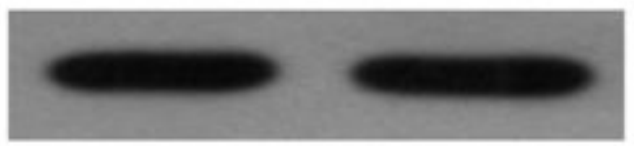

Figure 3

A. RNAi lentivirus vector sequencing map of RPL28 gene. B. Protein detection of exogenous and shRNA knockdown of RPL28. 

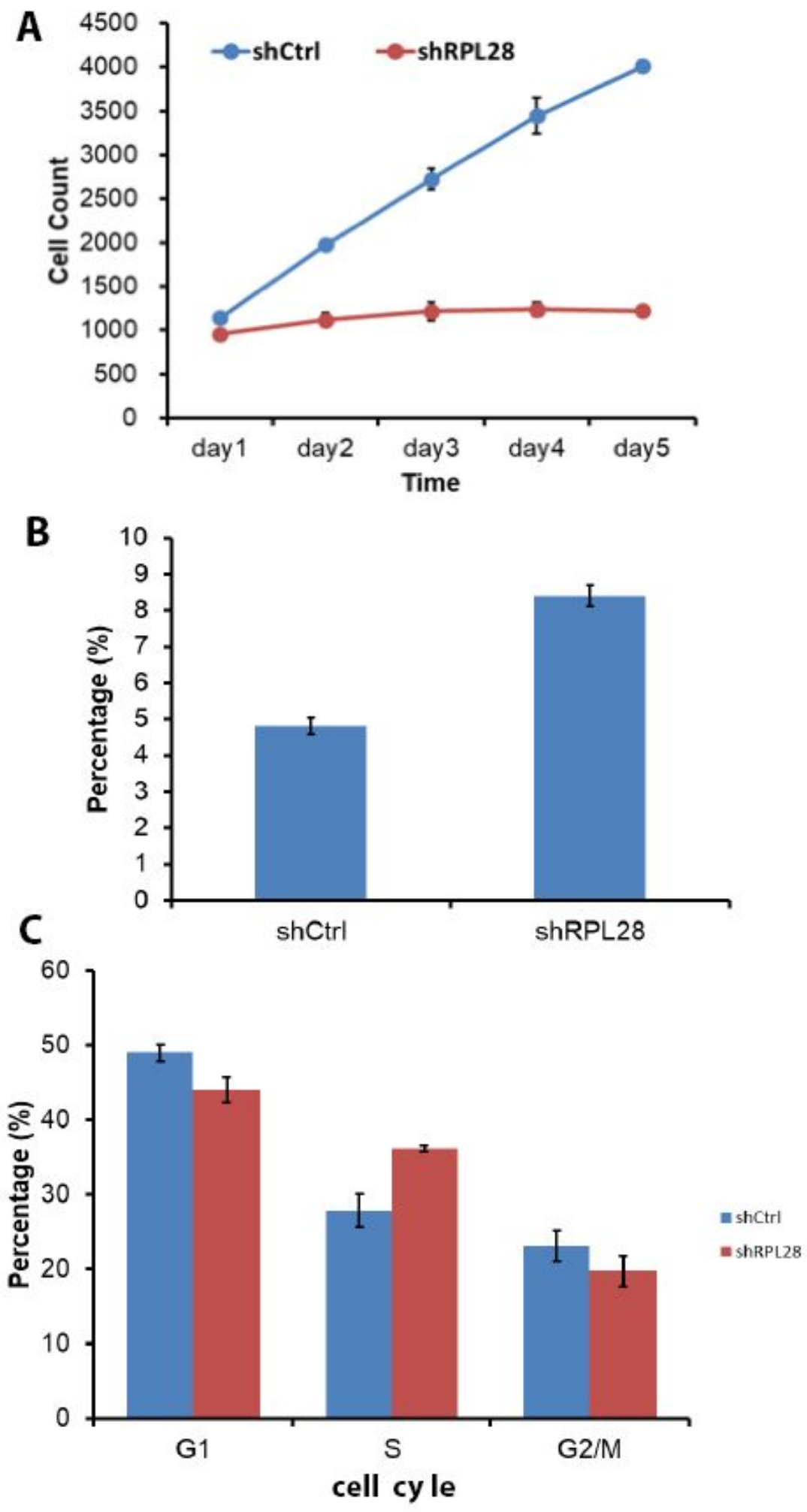

Figure 4

Cell counting, apoptosis and cell cycle analysis on shRNA lentivirus infected HepG2 sorafenib resistant cells. A. Cell counting result showed the proliferation of shRPL28 and shCtrl cells. B. Histogram of cell apoptosis $₫ \mathrm{X} \pm \mathrm{S} \bigotimes$ : The apoptotic rate of shRPL28 cells was higher than that of shCtrl cells. C. Cell cycle analysis result of $\mathrm{G} 1, \mathrm{~S}$ and $\mathrm{G} 2 / \mathrm{M}$ phase. 
A
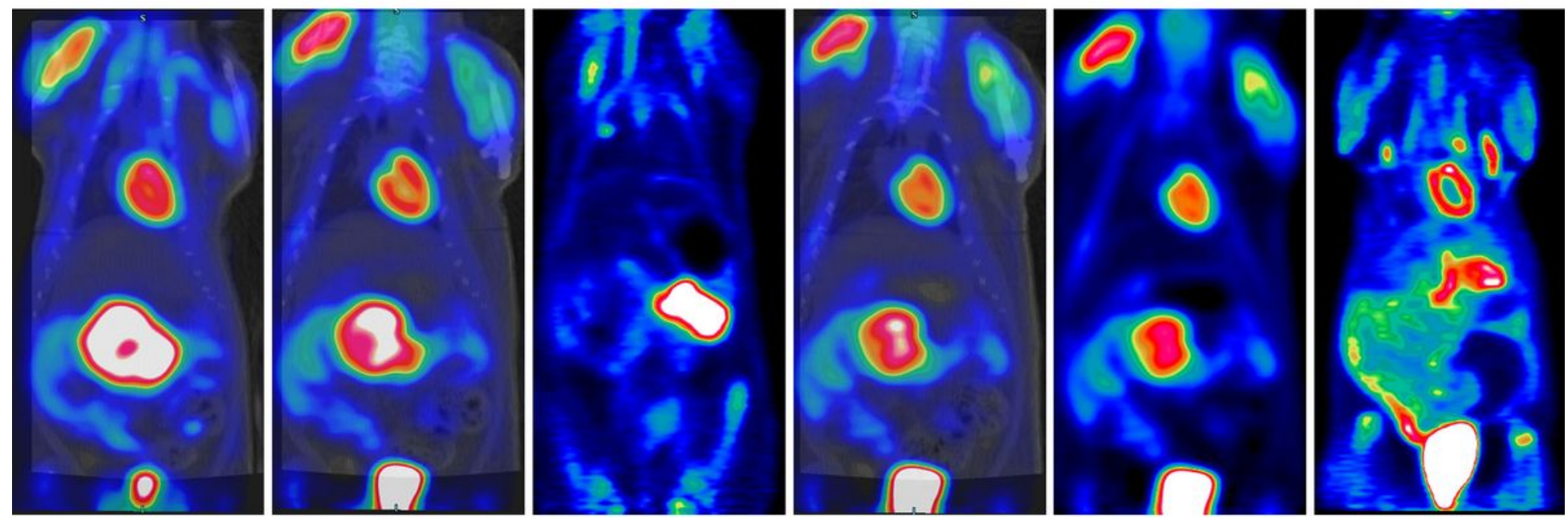

B
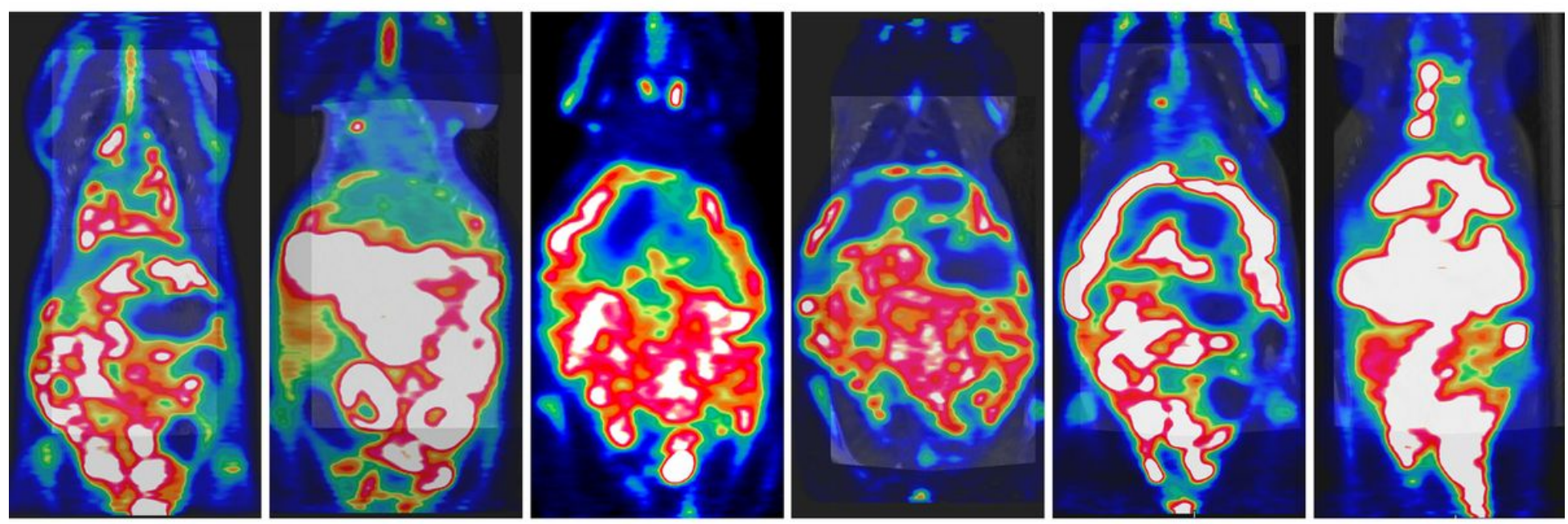

Figure 5

PET-CT of rats. A. Sorafenib sensitive stage: tumor growth was restricted. B. Sorafenib resistant stage: the tumor was widely metastasis to the abdominal cavity. 

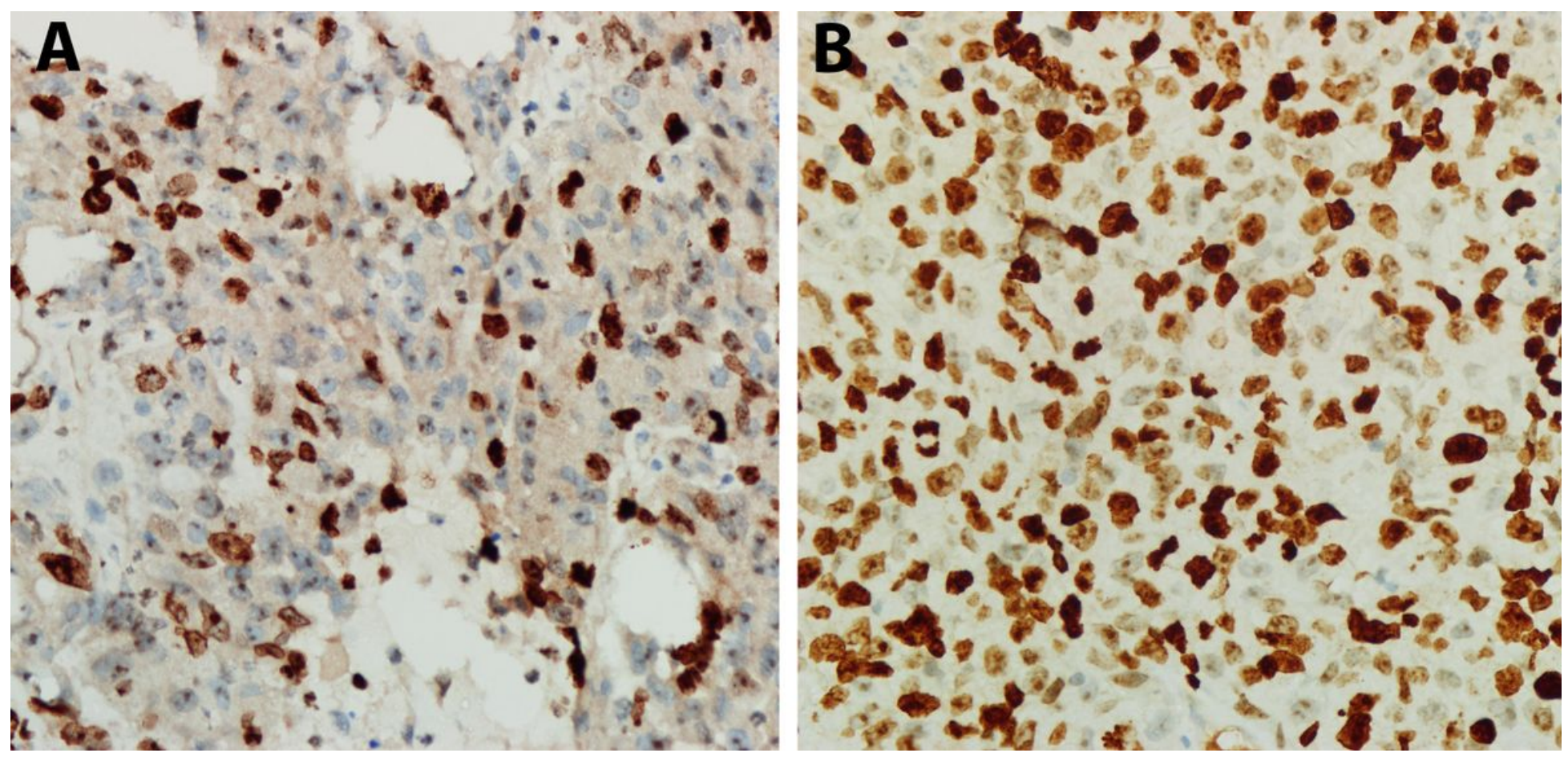

Figure 6

Expression of Ki67 in tumor specimens of sorafenib sensitive and resistant stage by immunohistochemistry (DAB developing, 400X). A. Sorafenib sensitive period, Ki-67 protein (Localization in nucleus) with low expression. B. Sorafenib resistance period, Ki-67 protein with high expression. 